\title{
Research and Implementation of key RFID technology in poultry products traceability system
}

\author{
Niu Xiang-jie ${ }^{1}$, Qu Yang ${ }^{2}$ \\ (1. School of Computer and Information \\ Engineering,Beijing University of Agriculture,Beijing \\ 102206, China 2.Beijing Academy of Agriculture and \\ Forestry Sciences ,National Engineering Research \\ Center for Information Technology in \\ Agriculture ,Beijing 100097, China)
}

\author{
Li Hua, \\ School of Economics\&Management, \\ Beijing University of Agriculture, \\ Beijing 102206, China (*Corresponding author)
}

\begin{abstract}
As an effective way to control the quality and safety of agricultural products, traceability system is aroused growing world attention. On the purpose of retrospective quality, this paper constructs a poultry production safety management and quality traceability system from IT view. The paper makes a detail analysis of the key RFID technology implementation process including the design principles of Write-reader hardware, micro controller module, power module and key RF module, also realizes the system quality retrospective features key technologies.
\end{abstract}

Keywords- traceability system; Quality safety of agriculture production; RFID technology

\section{INTRODUCTION}

In the recent years, the food safety crisis-such as livestock and poultry diseases, serious agricultural residual drugs and the increasing imports of foreign food materials occurs frequently, which caused widespread concern in the world[1 3]. How to effectively manage the production of livestock and poultry products is becoming an extremely urgent global issue. The mobile RFID technology is the emerging technology of RFID technical field, which through mobile devices and communication networks to realize the information exchange with RFID tag object[4 5]. Different from common RFID technology, the mobile RFID does not require wires to fix Write-reader, and the Write-reader installed on the mobile device, mobile phone or PDA in order to reduce the number of required Write-readers in certain coverage. Write-readers are connected to the wireless network or the Internet through mobile devices, and read the back-end database storage items labeled information. Currently, Organizations and associations such as NFC, ETSI and EPCglobal are starting the researches on mobile RFID. In addition, the technology combining RFID with IPv6 and LWAN network technology is the new direction of the future development of RFID technology. The paper applies RFID technology to poultry products traceability system, introduces details of the technical practice, and ensures that the needs of the poultry product traceability system.

\section{THE REALIZATION OF KEY RFID TECHNOLOGY IN POULTRY PRODUCTS TRACEABILITY SYSTEM}

\section{A. Control of write-reader in RFID system}

The write-reader is a significant part of the poultry product traceability RFID system, plays an important role in RFID systems. The write-reader primarily responsible for read or write tag data, get tag information, and then sent the information to the back-end system for processing. The reader is mainly composed with the control system and the radio frequency interface. Typically, control systems use a microprocessor and ASIC component to achieve its function.

The main functions of the write-reader control system are stated as follows: Implement command from application software, realize communication with application software, control the communication process of electronic tag and write-reader, realize encoding and decoding of the signal, implement anti-collision algorithm while the environment contains a large number of tags, encrypt and decrypt data to ensure security, and verify the write-readers and tags. Data exchange between the control system and the application software is completed through the interface of the reader and the host. The interface can be wired ( such as RS-322、RS485 or RJ-45) or wireless. The main functions of the highfrequency write-reader interface (RF module) are shown as follows: generate the high-frequency transmit power, provide work energy for the tags, modulate transmission signal and sent it to the electronic tags, receive and demodulate RF signal from the tag. Write-reader can send host command to the tag, also can send the obtain tag data to host, and what's more, it can control the data communication process. The particular functions are listed as follows:

(1) Communication functions of the write-reader and tags. According to the communication protocol to achieve communication with the tags and complete the information collection and data writing.

(2) Communication functions of the write-reader and the host. The write-reader can be connected to the host through a variety of standard interfaces, then upload the acquired poultry data to the host, or receive commands from the host. 
(3) The write-reader can operate multiple poultry tags in write-read zone, and correctly distinguish various poultry tags, mainly through the anti-collision algorithm.

(4) The write-reader can identify stationary and moving objects, make the application of RFID technology in a wider range and poultry data acquisition more convenient.

(5) The write-reader can prompt an error in the reading and writing process and display the error poultry information

(6) The write-reader can detect the battery information of active tags. The message will be issued in the case of low battery tags, in order to warn users to immediately replace the battery or tags to avoid error or malfunction caused by the lack of electricity.

\section{B. Hardware design of the write-reader}

The paper is the application in quality trace system with RFID technology. It designs a kind of high power, low power consumption poultry information Write-reader. The writer-reader can be divided into five parts-microcontroller module, RF module, PC interface module, storage module and power module, which can be seen in Figure 1.

The main function of the microcontroller module is to send commands to the tags, control the communication between the poultry tags and the writer-reader; it encodes the signal when sends the data; it needs plenty of storage space and high processing speed to run anti-collision algorithm in the environment with multiple poultry tags; it also encrypts and decrypt the data and identify the status between the writer-reader and tags in order to ensure the data security; it can read available data and upload to upper monitor when communicates with application software.

The RF module is the poultry data exchange interface between the writer-reader and electrical tags. The radio frequency carrier signal usually generated by the RF oscillator is processed by the RF processor, amplified by the $\mathrm{RF}$ amplifier and finally sent by the antenna.

The PC interface is used for the communication between the writer-reader and PC. The application software running in $\mathrm{PC}$ can send commands to the writer-reader via the interface. The writer-reader can also send the available data to PC by the interface. It will be convenient to process large amount of data in background to relieve the load of the Write-reader.

The data read by the Write-reader is processed at fixed time, thus the memory merely in the microcontroller can't meet the requirement for storage when the amount of data is large. The storage module will fix the problem. The module can store the data of the tags from the writer-reader whose volumes can vary according to the different applications.

The power module ensures the power supply of other modules and effective operation of the whole system.

\section{Micro-controller module}

In ETC system, the write-reader needs not only to run sophisticated anti-collision algorithm, but also to be able to read the tag data in the poultry identification process. Furthermore, the storage read label information requires a certain amount of memory, and environmental protection and energy saving are also cannot be neglected. Considering all the factors above, we choose high-performance and low consumption MSP430F149 to be the Micro-controller.

MSP430F149 has a CPU of 16 bits RISC structure and various addressing mode. With $125 \mathrm{~ns}$ instruction cycle, MSP430F149 integrates 16 general-purpose registers and constant generator that improve the encoding efficiency. It also supports downloading and online debugging. It's five power saving modes realize low power consumption, high stability and the perfect combination of high performance and ultra-low-power. Compare to other Micro-controller, the main advantages of MSP430F149 are shown as follow:

\section{1) Ultra-low power}

There are five power saving modes and one active mode. The current consumption is $280 \mu \mathrm{A}$ under the operating frequency and voltage of $1 \mathrm{MHz}$ and $2.2 \mathrm{~V}$ in the active mode, and $1.6 \mu \mathrm{A}$ in standby mode, $0.1 \mu \mathrm{A}$ in power down mode.

2) Strong processing capacity

MSP430F149 has various addressing capacity. Multiple (16) on-chip registers make it easy to achieve a variety of operations, and ensure the operating efficiency of the anticollision algorithm.

\section{3) High instruction execution speed}

Under $8 \mathrm{MHz}$ clock frequency, MSP430F149 has a $125 \mathrm{~ns}$ instruction cycle. When the system is in a power saving mode, we only need as long as $6 \mu$ s to wake-up the MCU using the interrupt request.

4) Large storage space

MSP430F149 microcontroller has built-in $60 \mathrm{~KB}+256 \mathrm{~B}$ Flash and 2KB RAM. In MSP430 system, the RAM is large.

There are also 16-bit timer for system internal clock, four 8-bit parallel ports which rich I / O functions, and two serial communication interface which can easily achieve communication with the host.

\section{RF modules}

RF module uses nRF24L01 which is a single-chip RF transceiver device working in $2.4 \mathrm{GHz} \sim 2.5 \mathrm{GHz}$ globally open ISM band. Its operation rate can be up to $2 \mathrm{Mbps}$. It uses GFSK modulation and includes $125 \mathrm{RF}$ communication channels in which each channel can be configured through the process to satisfy the multi-point communication needs; additionally, the chip contains a frequency synthesizer, a power amplifier, a crystal oscillator, modulator function module, built-in hardware CRC error detection and point-tomultipoint communication address controller. The power consumption of the chip is low-the electrical current is $22 \mu$ $\mathrm{A}$ in standby mode and $900 \mathrm{nA}$ in power-down mode. The voltage is $1.9 \mathrm{~V} \sim 3.6 \mathrm{~V}$. The chip has very strong antiinterference ability. The scheme of the RF module is shown in figure 2.

nRF24L01 module has two communication modes: ShockBurstTM (Burst mode) and Enhanced ShockBurstTM (Enhanced Burst mode).

While using ShockBurstTM communication mode, the microcontroller doesn't need high-speed data processing and timer reset. nRF24L01 can utilize the interior first-in-firstout stacks to finish the high-speed(up to $2 \mathrm{Mbps}$ ) data 
transmission. It can increase the transmission rate in RF link as larger as possible with decreasing the average current consumption when the microcontroller runs in low-speed situation. In this mode the $1 \mathrm{Mbps}$ communication rate can be compatible with nRF2401, nRF240, nRF24E1, nRF24E2. When it sends something, the hardware will automatically add the address and CRC checksum then inform the complete of send by interrupt occurring in order to decrease the need of microcontroller storage which can decrease the cost and shorter the software development time. When it receives something, the interrupt will notify the microcontroller the received available data. Then the microcontroller can read the data from RX_FIFO.

Enhanced ShockBurstTM mode has embedded communication protocol to make duplex link communication much simpler and more effective except with the normal functions of ShockBurstTM mode. For typical duplex communication, the receiver will send ACK to the sender to notify the data is received when it receives the data. The sender will judge whether the send is successful or not by detecting the ACK. The sender will resend the data if the previous one is failed. In Enhanced ShockBurstTM mode, nRF24L01 will send ACK and resend the data without the participation of the microcontroller. When nRF24L01 is as the receiver, it can use 6 data channels to receive data and each data channel has the sole address which means it can receive data from 6 devices simultaneously, and then it will send ACK with the address of received data. When nRF24L01 is as sender, it has the same characteristics with ShockBurstTM mode.

No matter the ShockBurstTM mode or Enhanced ShockBurstTM mode, it will save the resource of the microcontroller and make the microcontroller work more efficiently. It has many advantages: sending RF signal in high speed, strong anti-interference ability; lower the average current in the whole system. Especially in ShockBurstTM mode, it can be compatible with nRF2401, nRF2402, nRF24E1 and nRF24E2 to communicate with multiple tats, which make the writer-reader has more application situation.

\section{E. Storage Module}

The writer-reader sends the poultry information daily. If the storage space is full, the writer-reader will send the data to background or it will send the data at fixed time. Because of the operation mode, the poultry information isn't sent to background processing center real-time. The storage devices need to save the poultry information read.

Considering one card for one poultry, the writer-reader need to store the number of the tags then the number is processed background. The number of the tags is represented in two hexadecimal numbers thus the storage space needs two bytes. In order to let the users know the tags, the time read every time is stored including hours, minutes and seconds which takes 6 bytes. AT24C64 is used to temporally store the data collected. AT24C64 is from Atmel and the storage space is $64 \mathrm{Kbit}$. According to set data format, 8 bytes is needed to store a tag information. Thus AT24C64 can store 1000 poultry information. When the amount of the poultry information is large, AT24C128/256 can be used to increase the storage space.

\section{F. Interface module}

The communication mode between the writer-reader and $\mathrm{PC}$ is serial. PC writes programs or sends commands to the writer-reader via serial port. The writer-reader can also send tag information to PC by serial port. This design uses MAX232 from MAXIM. It can work normally under the voltage of $3 \mathrm{~V} \sim 5.5 \mathrm{~V}$. The interface is 9-points D-type socket. T1IN and R1OUT connect the serial receiver and sender of the microcontroller respectively. T1IN and R1OUT connect the second and third points respectively. The chip has very simple peripheral circuit and wide application. The typical application scheme is as shown in figure 3 .

\section{G. Power module}

The whole writer-reader applies 5V power. LP2951$30 \mathrm{DG} 4$ three terminal regulator will output $3 \mathrm{~V}$ power to the device. LP2951-30DG4 three terminal regulator is high costeffective regulator suitable for battery. It has wide voltage output range whose highest is $30 \mathrm{~V}$ and fixed output voltage is $5 \mathrm{~V}, 3.3 \mathrm{~V}$ and $3 \mathrm{~V}$. In the environment of $25^{\circ} \mathrm{C}$, the output voltage error is $0.5 \%$. The device has very good line and load regulation rage. When the input voltage is 5V, LP2951$30 \mathrm{DG} 4$ can output $3 \mathrm{~V}$ to provide work voltage to other devices. The scheme of the power module is as shown in figure 4 . The filtering capacitors are connected to the output and input end of the there terminal regulator in order to make the current smoother and the voltage stable.

\section{ACKNOWLEDGMENT}

Chinese book Classification Code: TP391.9 Document code: A

\section{CONCLUSION}

On the purpose of retrospective quality, this paper constructs a poultry production safety management and quality traceability system from IT view. The paper makes a detail analysis of the key RFID technology implementation process including the design principles of Write-reader hardware, micro controller module, power module and key RF module, also realizes the system quality retrospective features key technologies.From the experiment results, the system can increase the enterprise production management level and enhance the safety awareness of the agriculture products for the consumers, build solid base for poultry full process trace.

\section{REFERENCES}

[1] Schwagele,F. Traceability from European perspective[J].Meat Science, 2005(71):164-17

[2] MousaviA,SarhadiM,LenkA,eta1.Tracing and traceability in the meat processing industry: a solution[J].British Food Journal, 2002, 104(1) : 7-19.

[3] SmithGC,TatumJD,BelkKE,etal. Traceability from aUS perspective[J].Meat Science, 2005, (71) : 174-193. 
[4] StanfordK,StittJ,KellarJA,etal. Traceability in cattle and small ruminants in Canada[J].Scientific and Technical Review, 2001, 20(2) : $510-52$
[5] BlancouF.A history of the traceability of animals and animal product[J].Revue Scientifiqueet Technique, 2001, 20(2) : $413-$ 425
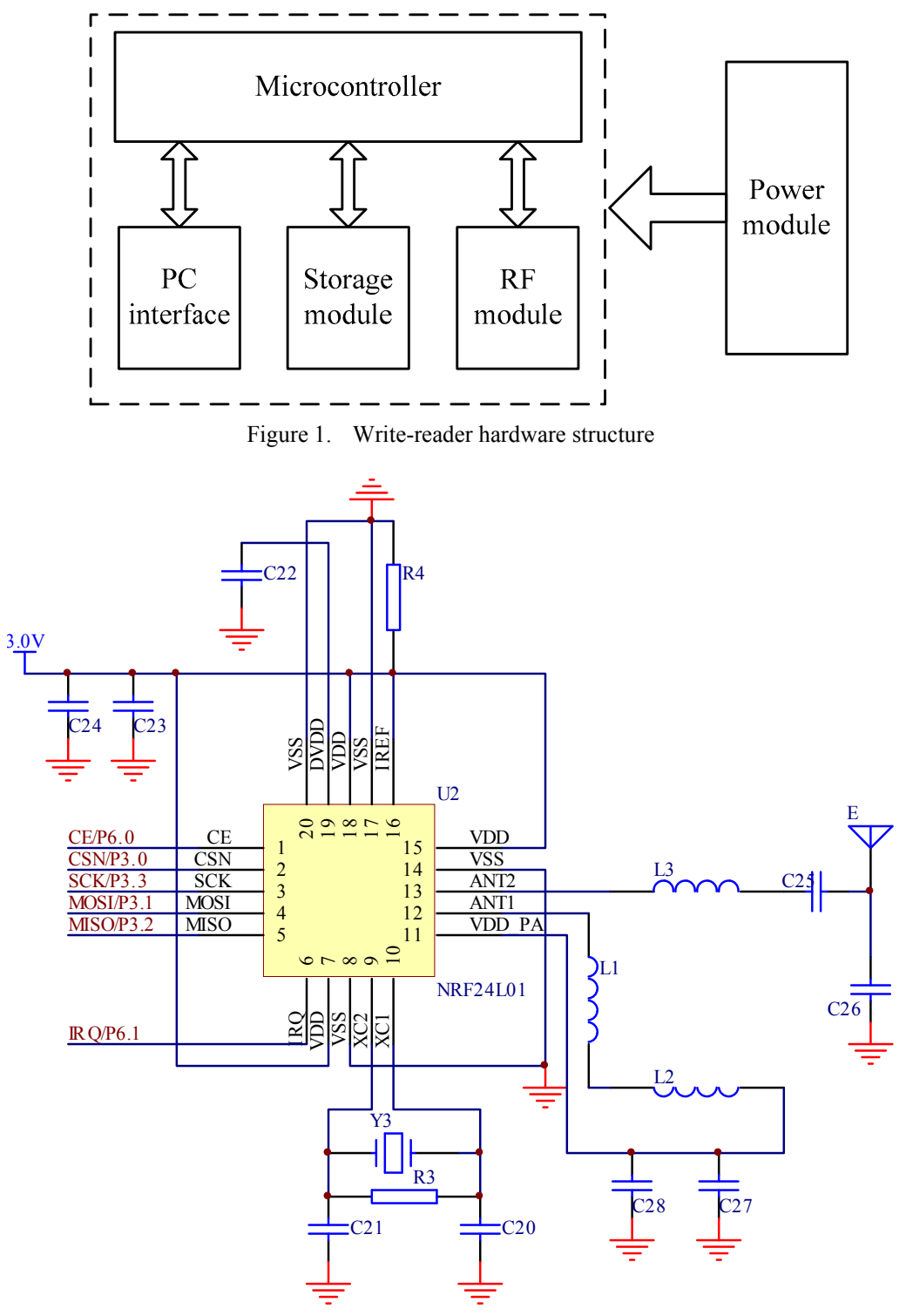

Figure 2. RF module electrical scheme 


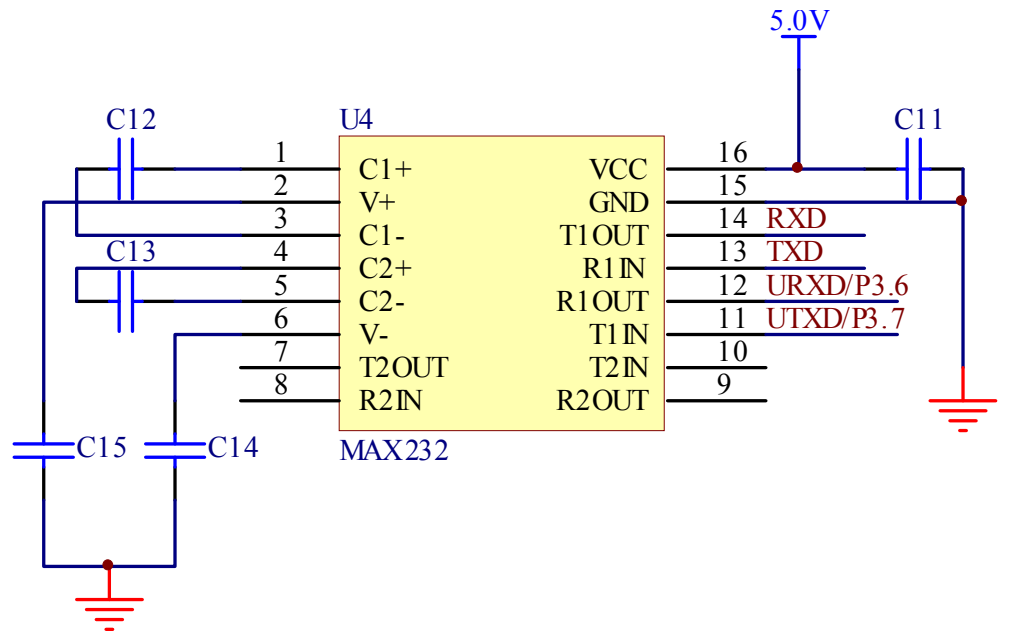

Figure 3. PC interface module scheme

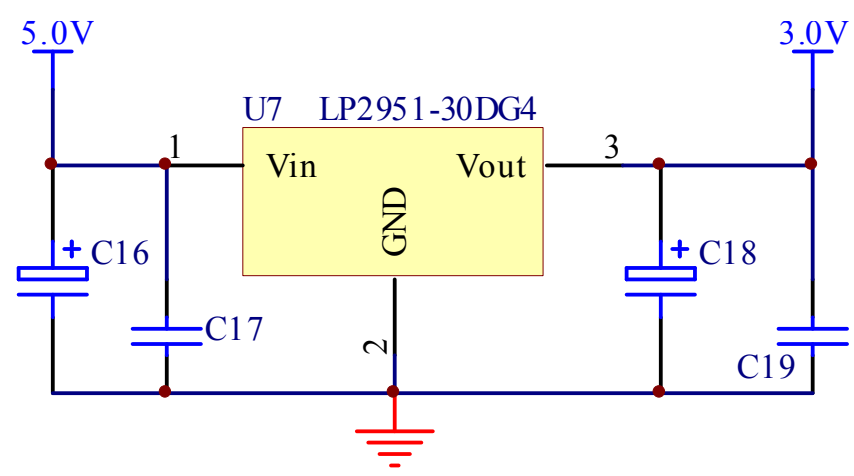

Figure 4. Power module scheme 